\title{
Optimized Software Licensing - Combining License Types in a License Portfolio
}

The increasing number of diverse concepts and pricing models for software licensing complicates the allocation of the license portfolio at optimal cost. License types are combined considering the users' prespecified demand and availability requirements. Optimal licensing for a particular group of users can be estimated without detailed knowledge of the individual user behavior, of group size, and the cost ratio between single user and network licenses. In the case of multiple heterogeneous user groups, however, optimal licensing of use-intensive applications can only be carried out considering group-specific capacity profiles, due to compensation effects. In order to cover cost-intensive peak loads and reduce risk of service quality of network licenses or load fluctuations simultaneously, it is possible to additionally use on-demand licenses, whose offers are still limited to only a few types of application.

DOI 10.1007/s12599-009-0063-2

\section{The Authors}

\author{
Dipl.-Inf. Daniel Gull \\ University of Augsburg \\ FIM Kernkompetenzzentrum \\ Finanz- \& Informationsmanagement \\ Universitätsstr. 16 \\ 86135 Augsburg \\ Germany \\ daniel.gull@wiwi.uni-augsburg.de \\ Dr. Alexander Wehrmann \\ Senacor Technologies AG \\ Vordere Cramergasse 11 \\ 90478 Nürnberg \\ Germany \\ alexander.wehrmann@senacor.com
}

Received: 2008-03-10

Accepted: 2009-01-19

Accepted after two revisions

by Prof. Dr. Hasenkamp.

This article is also available in German in print and via http://www.wirtschaftsinformatik.de: Gull D, Wehrmann A (2009) Optimierte Softwarelizenzierung - Kombinierte Lizenztypen im Lizenzportfolio. WIRTSCHAFTSINFORMATIK. doi: 10.1007/11576-009-0182-x.

\section{Introduction}

According to a recent study by Mendel and Takahashi (2007, p. 6), software licenses and license maintenance agreements constitute the third largest item of many European companies' IT budget with $14 \%$, following hardware (19\%) and personnel (29\%) cost. Compared to self-developed software, management of software subject to a license constitutes a challenge for every company.

An insufficient license management can result in costly over licensing, when unnecessary licenses are acquired. A study by Gartner (2001) assumes that $80 \%$ of the top 500 companies are affected in this way. Meta Group (CIO 2003) estimates that unused software in companies amounts to $\$ 9$ billion worldwide. KMPG (2002, p. 9) and Müller et al. (2006, p. 15) see a practicable potential for cost reduction of up to $15 \%$ by an optimized license management. The problem of over licensing is further intensified by the predominating legal insecurity with regard to reselling licenses. The usual procedure of buying license proofs instead of full versions with data media may in the end turn out to be a cost trap for companies.

Besides penal consequences, under licensing may lead to serious claims for indemnification by the manufacturer.
Nevertheless, about $28 \%$ of the software in Germany is unlicensed according to IDC (2007, p. 4). Although this seems moderate compared to eastern European and Asian countries, such as e. g. Armenia (95\%) or China (82\%), software producers have become sensitized. A media company has recently been sued by the Business Software Alliance (BSA), funded by the software industry, and convicted to 2.5 Million Euros compensation (BSA 2007).

According to a study commissioned by Microsoft (2007, p. 5), there is still considerable need for additional information regarding license management. The study states that only $44 \%$ of the interviewed companies check for under or over licensing.

A legally correct and simultaneously economically efficient licensing not only requires to determine the optimal number of licenses, but also to choose the right type of licensing. The increase of global networking as well as the tendency towards more flexibility by means of virtualization and on-demand services, such as cloud computing or software as a service (SaaS), offers the possibility of providing and licensing enterprise systems and applications in a great variety of ways. New licensing models are usually accounted for per use, which is e. g. measured by counting the minutes or function 
calls, or per value. Besides classical models, some producers now also offer prepaid rates with an accordingly limited risk of cost. In addition to local workplace installations and the provisioning via terminal services or virtual desktop systems, online applications play an increasing role. These are usually offered directly by the manufacturer, play an increasing role (a typical representative would be the web application "HR-works" for travel expense accounting or the widely used conference platform "WebEx").

The number of different licensing type offers is steadily growing, ranging from static single-user licenses to use-dependent on-demand licenses. Finally, the combination of different license types not only has an impact on the license costs, but also affects the cost risk. Cost risk means that the licensing costs for the considered period differ from the expected value. This risk directly reduces the planning security of the IT budget, which is important especially in times of tight IT budgets.

This article shows how in the course of license portfolio management different license types should be combined in an optimal way, taking into account costs, cost risk, and guaranteed quality of service. In this context, quality of service describes the degree of availability or the user's waiting time when calling the software if only a limited number of parallel accesses are possible. We show that by means of an optimized license portfolio management, significant savings may be achieved. The introductory chapter is followed by a fundamental optimization model with single user and network licenses which then will be extended by on-demand licenses. The paper ends with a summary and an outlook. The accompanying examples will demonstrate the practical relevance of the model.

\section{Status quo of license portfolio management in research and practice}

License management covers all tasks related to the use of software licenses. The relatively few existing contributions on this specific topic are primarily dedicated to the management and distribution of licenses in the company, without explicit recommendations for the selection of appropriate license types. Wisotzky (2006, p. 6), for example, classifies license management into contract management, inventory, usage measurement, gap analysis, contract optimization, dwelling especially on the benefit of automating these tasks. Bensberg and Reepmeyer (1994, p. 595) show how such an automated license management system can be implemented, giving the example of a local network at the University of Münster.

Müller et al. (2006, p. 17), however, in particular include financial management in an economic license management. In the context of software license agreements, manufacturers may not only grant advantageous payment conditions, but also offer discounts for bundled quantities. All parts of the license management, based on the targets licensing costs and cost risk, can be assigned to the financial management. Therefore contractual possibilities as well as the measurement and analysis of software usage play a role for the optimization.

Surprisingly, there is yet little literature which addresses the question on how to assemble an economically advantageous license portfolio of different types of licenses. Znidarsic (2006) takes up the issue of license types, but does not dwell on a concrete optimization. Järvinen et al. (2007) perform a quantitative analysis to determine the optimal number of network licenses. As stated by the authors themselves, the approach is based on the somewhat unrealistic assumption that the frequency of use by a group of users remains unchanged over the whole period examined. As we will show later, this assumption is without problems only for calculating the optimal number of network licenses with just one group of users. In case of more user groups coinciding during the given period or in case of using on-demand licenses, the presented approach by Järvinen et al. does not live up the expectations. Moreover, the question remains unsettled when a combination of single user, network, or on-demand licenses for several groups is economically efficient and how the quality of service risk can be reduced, i. e. how to ensure that the assured availability of the application is respected. This paper will include closing these gaps in the optimization of the license portfolio.

\section{License portfolio management}

The aim of license portfolio management (LPM) - as part of license management
- is the demand-oriented selection of appropriate license types and their allocation to a license portfolio. In the following we present an optimization model which allows for objectifying decisions regarding the economic design of license portfolios taking into account cost and cost risk issues. This also considers the available license types, the user-specific intensity of use, and the required quality of service. These three aspects will be briefly explained first.

\subsection{License types}

Software can either be purchased, i. e. the buyer receives the property rights, or be used as part of software licensing (see Stapperfend 1991, p. 87-94; Buhl 1993, p. 913). Purchasing a software license (lat. licere $=$ permit) simply includes the right to use a copy of the software (see Sedlmeier 2006, p. 10). In the case of traditional licensing, i. e. the purchase of the license, the runtime is unlimited (perpetual). In the case of license rents (subscription), the right of use is limited in time. A study by Macrovision (2006) showed that in 2005 already $40 \%$ of the 256 surveyed software manufacturers offered subscription licenses. The offer of perpetual licenses, however, has already been reduced by $7 \%$ from 2004 to 2005 and is expected to continue to decrease in the years ahead.

In addition to the runtime, the license regulates the type of licensing. In general, we can distinguish two types: Static license types are directly bound to systems or users and thus cannot be redistributed or shared at short notice. The license conditions can be defined as follows: the number of licenses must correspond to the number of installed systems - in rare cases users - or a transfer ("reassignment") after a certain period (e. g., 90 days) is possible. In the case of firmly assigned "named user/client" licenses, however, a transfer usually is only allowed in accordance with the manufacturer. Dynamic license types may be flexibly reallocated at any time if required, or in the case of on-demand licenses may either be requested ad hoc or expended successively. In this case, gradual consumption may also be accurately determined offline, for example by means of a hardware dongle.

The type of application provision is largely independent from this issue. In general, both for locally installed appli- 


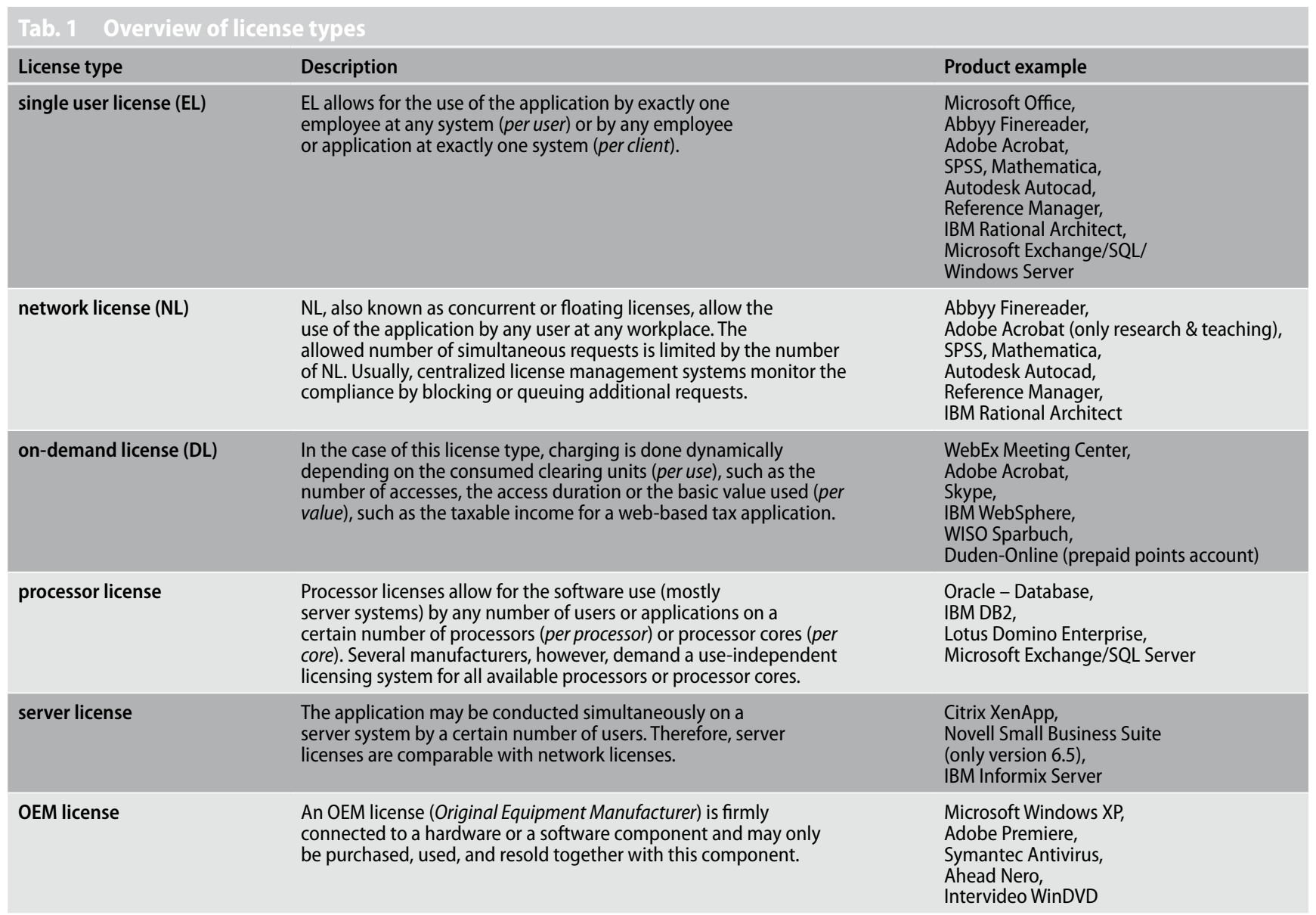

cations and for centralized application platforms, such as e. g. web applications, the use of all the license types mentioned above is possible. Tab. 1 provides an overview of the most commonly used license types. The availability of a license type may change with a product version change (e. g. Novell Business Suite) or may be limited to specific user groups, such as research and teaching institutions (e. g. Adobe Acrobat).

The trend towards more flexibility and demand orientation increases the range of license types. EL, which still were preferred by the majority of customers in 2004 , had already fallen far behind network licenses by 2005. Manufacturers are also beginning to rely on on-demand models and thus plan to expand their offers within the next few years (Macrovision 2006). In the following optimization model we therefore consider the first three license types, whereas the results can basically also be transferred to other license types (e. g., processor licenses).

\subsection{Intensity of use}

For new software, the estimation of licensing demand can be carried out based on the manufacturer's experience data, user surveys, or the analysis of existing data. On the basis of transactions, process cycles, or documents that need to be prepared, conclusions about the expected intensity of use can be drawn. For the replacement of existing or comparable applications, ideally data from a software metering exist. Software metering systems log the period-related number and duration of accesses to applications and not only provide a detailed analysis of the use intensity, but also allow for the differentiation of users according to their usage behavior. If only static licenses can be used, it is sufficient to determine the maximum number of different users or systems. In case of dynamic licenses, however, the intensity of use plays a crucial role. As the collection of personal data is only possible under certain conditions pursuant to $\$ 4$ of the German Federal Data Protection Act (BDSG 2003), software metering usually requires the consent of each employee or the employee representatives (e. g. works councils). Therefore, this possibility of analysis is ruled out for some companies.

From the (average) use frequency and duration we can deduct the intensity of use for any time interval (referred to as license load in the following) and calculate it for a specific course of time (e. g. day or month) as a load profile. The optimization model does not specify a fixed frequency of time intervals. As granularity affects accuracy, however, and thus has a direct impact on the outcome, we have to determine the interval size in every single case on the basis of conditions, such as period, measurement effort, etc.

In the case of a dimensioning of network and telecommunications equipment, the load profile is usually divided into constant intervals of 60 minutes each. The time interval of the highest load is called the main traffic hour or busy hour (BH) (see Cole 1998, p. $386 \mathrm{ff}$ ) This interval affects the quality of service since at this time the most resources are needed.

Fig. 1 shows an example of the load profiles of two user groups on a working day. Group A has its BH between 10:00 and 11:00 a.m., Group B between 13:00 and 14:00 a.m. The license load (intensity) 


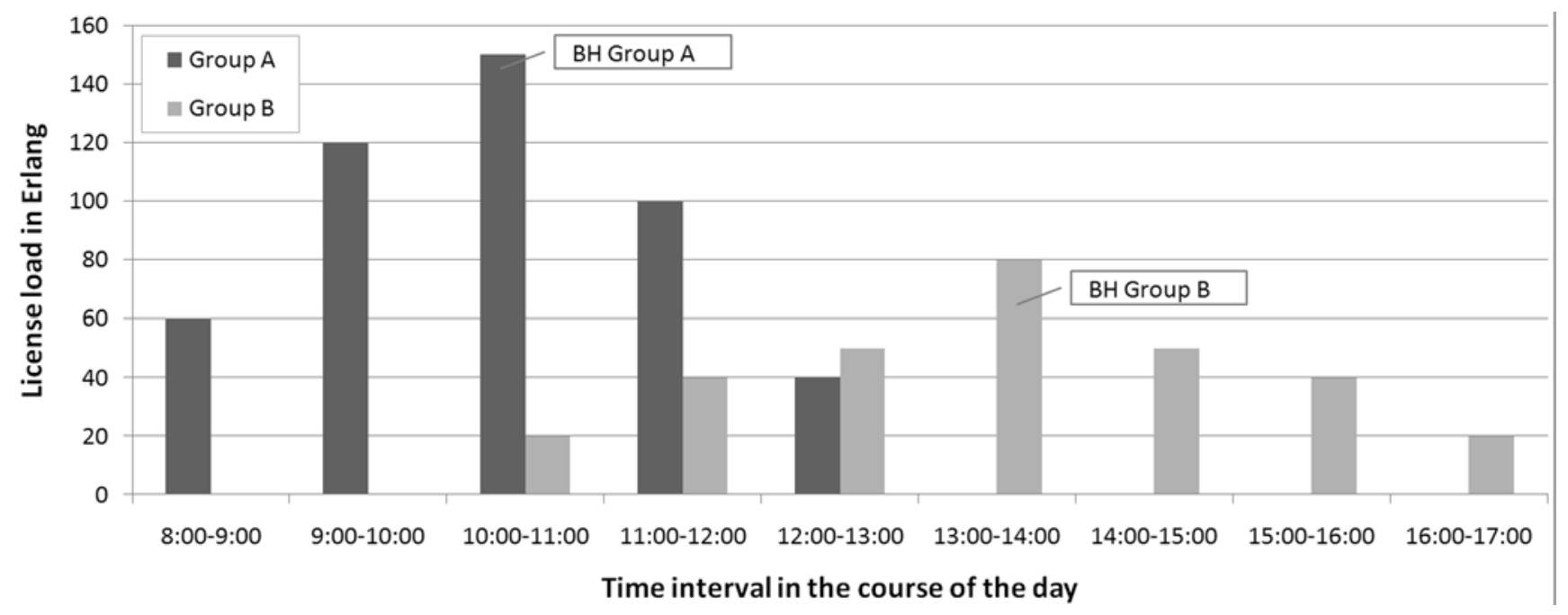

Fig. 1 Load profiles of two user groups

per time interval is measured based on the dimensionless measurand Erlang (E), named after the mathematician Agnus Krarup Erlang (ITU-D 2005, p. 40). The license load is the product of the use intensity and the use duration per unit of time:

license load $=\frac{\text { use frequency.use duration }}{\text { time unit }}$

One Erlang corresponds to the use of exactly one software license if all requests take place without interruption in sequential order. If there are not enough licenses for software applications available, not all requests can be served immediately. The result is a reduced quality of service, which may have different characteristics depending on the licensing procedure.

\subsection{Quality of service}

A central licensing allocation corresponds to an operating system with a stochastic arrival and adjustment process. These systems, also known from queuing theory, are classified with the notation $(a / b / c):(d / e / f)$ which has been introduced by David G. Kendall and later extended by Lee and Taha (1992, p. 554 ff). A and b characterize the probability distribution of the arrival and service process, with the letter M (markovian) standing for poisson or exponentially distributed, G (general) for generally distributed, and D (deterministic) for constant. The parameter $c$ stands for the number of service units (here: licenses), $d$ for the total capacity of the system including queue, e for the number of system users, and f describes the service discipline. If $\mathrm{d}, \mathrm{e}$, and $\mathrm{f}$ are not specified, $\infty / \infty /$ FIFO (first-in-first-out)

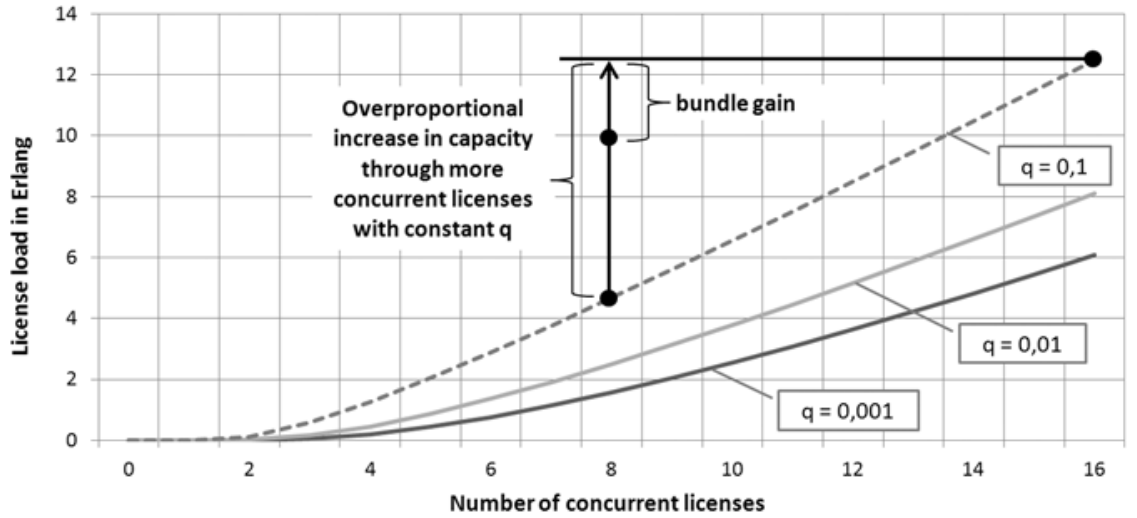

Fig. 2 Load capacity of network licenses with constant quality of service level

holds for these parameters (see Stache and Zimmermann 2001, p. 361-384). In the case of a license without queue $(\mathrm{d}=\mathrm{c})$, requests are removed (blocked) from the system as soon as no more licenses are available. The quality of service here corresponds to the blocking probability. If an application call is not rejected but added to the line $(\mathrm{d}>\mathrm{c})$, there is a waiting system and the quality of service can be determined based on the average waiting time (Rey 1983, p. 148 ff) Therefore, the quality of service is an essential part of the optimization for central licensing.

\section{Models for optimizing the license portfolio}

First, we present a basic model which allows for optimizing license costs for one user group, including only single user (EL) and network licenses (NL). Subsequently, we shall expand the model to multiple user groups and on-demand licenses (DL).

\subsection{Single user and network licenses for one user group}

The basic model is based on the following assumptions:

(A1) For a group with $A_{G e s} \in \mathrm{N}$ users, a license application subject to licensing is provided.

(A2) For licensing, single user licenses (EL) as well as network licenses (NL) are available. The allocation of the NL is made either by a loss system or a waiting system. In this case, $A_{E L} \in \mathrm{N}_{0}$ corresponds to the users who receive the EL and $A_{N L} \in \mathrm{N}_{0}$ to those users who receive an NL.

(A3) For the user group, the average load profile $P$ with $T$ time intervals is known. This contains for each time interval $t \in T$ with the expected length $z_{t} \in R^{+}$the expected intensity of use $n_{t} \in \mathrm{N}_{0}$ and the expected use duration $d_{t} \in R^{+}$. The arrival 


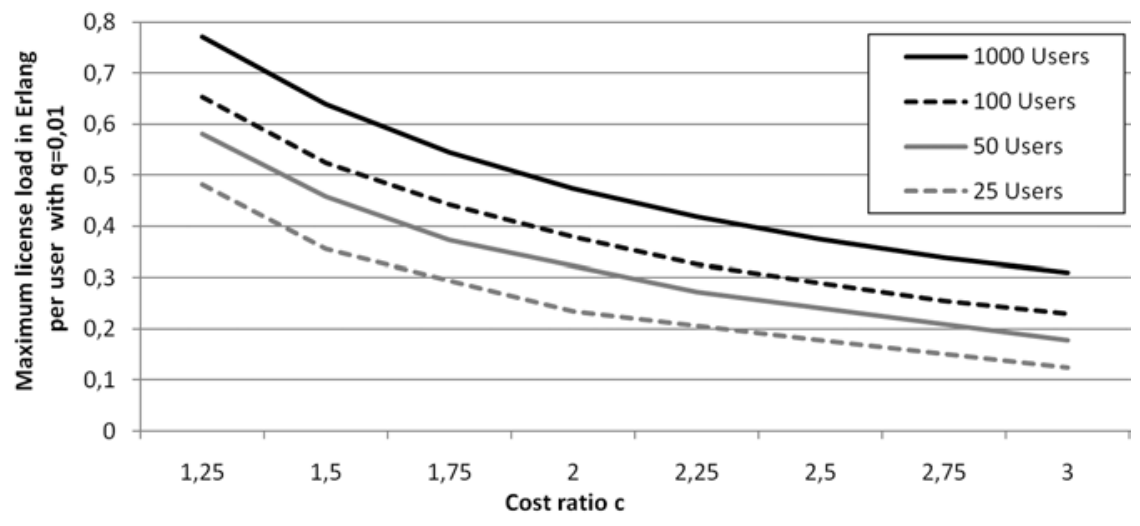

Fig. 3 Maximum user load for different cost ratios and group sizes

rate $\lambda$ in the time interval $t$ is $\lambda_{t}=\frac{n_{t}}{z_{t}}$, the expected operating time $\mu_{t}=\frac{\tilde{I}^{t}}{d_{t}}$. $L_{t}(P)=\frac{n_{t} \cdot d_{t}}{z_{t}}=\frac{\lambda_{t}}{\mu_{t}}$ corresponds to the license load (in Erlang) of the load profile $P$ in the time interval $t$. The time interval with the highest load is referred to as $\tau$ (busy hour). The greatest license load (busy hour traffic, BHT) is: $L_{\tau}(P)=\frac{\lambda_{\tau}}{\mu_{\tau}}$.

(A4) For a loss system, $q_{t}=B\left(L_{t}(P), N_{N L}\right)$ is the probability that for $N_{N L}$ available NL a request is rejected (blocked) in the time interval $t$ of the load profile $P$. For a waiting system, $q_{t}$ corresponds to the expected waiting time until a license is assigned. The highest blocking probability or waiting time in $\tau$ is $q_{\tau}=B\left(L_{\tau}(P), N_{N L}\right)$. Thus, the resulting quality of service $q$ of the system is: $q=q_{\tau}=B\left(L_{\tau}(P), N_{N L}\right)$. For the calculation of $B$, we can make use of various functions from queuing theory, depending on the allocation method (Bose 2002, p. 9-23).

(A5) The minimum number of NL for a given load and given quality of service $Q$ is determined by $S\left(L_{t}(P), Q\right) \in N_{0}$, which is an inverse function of $B\left(L_{t}(P), N_{N L}\right)$. $N_{N L}=S\left(L_{\tau}(P), Q\right)$ corresponds to the number of NL to uphold the quality of service $Q$ in the load profile $P$ at every point in time. In the case of complex $B$ (e. g. Erlang formula), $S$ may be approximated numerically or determined by tables (see Junk and Warnecke 2002, p. 109). Basically, the determination can also be based on simulation, as e. g. Järvinen et al. (2007) show.

(rAf). EL or NL cause constant license costs in the amount of or $K_{E L} \in R^{+}$or $K_{N L} \in R^{+}$, where $K_{N L}=c \cdot K_{E L}$ is valid with $c \in R^{+}$and usually $c>1$. The total costs $K_{G e s} \in R^{+}$for the application correspond to the single user license costs multiplied by the number $N_{E L} \in N_{0}$ and $N_{N L} \in N_{0}$ of required EL and NL. There proportional $u, v=1$ are com- mon due to usual volume discounts in addition to declining cost functions with $0<u, v<1$. Only license costs are considered. Additional costs or savings that may be caused by the use of a license type (e. g. administrative costs) are not included in the first instance.

$$
\begin{gathered}
K_{G e s}=K_{E L} \cdot N_{E L}^{u}+K_{N L} \cdot N_{N L}^{\nu} \\
K_{G E S}=K_{E L} \cdot\left(N_{E L}^{u}+c \cdot N_{N L}^{\nu}\right)
\end{gathered}
$$

(A7) The objective is to reduce the overall license costs $K_{G e s}$ to a minimum. Moreover, the additional condition requires that the resulting quality of service $q$ does not exceed the given quality of service $Q$ in the load profile $P$ :

$$
\begin{aligned}
& K_{G E S} \rightarrow \text { Min } \\
& \text { under the condition: } q \leqslant Q
\end{aligned}
$$

Similar to a data or mobile network with a limited number of channels, an operating system for NL has the following property: A larger number of NL may handle disproportionately more license load at a constant quality of service, since the random (not strictly sequential) requests with an increasing number of NL can be distributed efficiently, thus disproportionately increasing the probability of a (faster) allocation. This effect, which is reflected in the non-linear course of the quality of service curve, can be explained only qualitatively (see Tran-Gia 2005, p. 93) and is usually referred to as directive gain or economy of scale (Junk and Warnecke 2002, p. 109).

Fig. 2 displays three curves, each one with a constant quality of service level (here: blocking probability). The dashed curve $(q=0,1)$ shows that in case of a doubling of the NL from 8 to 16 , the capacity is more than doubled (from under 5 to over 12).

\section{Homogeneous users groups}

If no distinction is made between the users in a group and therefore they are considered homogeneous in terms of their behavior, an exact determination of the optimal licensing with EL or NL under the above assumptions can result directly. The effect of the directive gain shows that in case of an increasing number of users $\left(A_{N L}\right)$ with the same (homogeneous) user behavior, the number of required NL $\left(N_{N L}\right)$ rises only in a slighter degree and not proportionally. For instance, also the analytical cost model for broadband networks by the regulatory authority for telecommunications and posts is based on this characteristic (RegTP 2005, p. 23). In contrast, the number of required EL $\left(N_{E L}\right)$ is always directly proportional to the number of users $A_{E L}$. Consequently, the linear combination of the two cost functions is concave, and hence the minimum is always located at the edge. The optimum of the total cost is equal to the minimum of the cost of a full licensing with EL or NL for the load profile $P$ and the quality of service $Q$ :

$K_{G e s}^{*}=K_{E L} \cdot \operatorname{Min}\left\{A_{G e s}^{u} ; c \cdot N_{N L}^{\nu}\right\}$ with $N_{N L}=S\left(L_{\tau}(P), Q\right)$.

By means of the function $S$, it is possible to determine the optimal number of required NL. In the case of load variations, however, there is a risk with regard to the availability of the application, so that for high availability requirements a correspondingly smaller value for $Q$ has to be specified. The optimum is independent of $K_{E L}$ and $K_{N L}$ and depends only on the cost ratio $c$ of both sizes.

\section{Non-homogeneous user groups}

A homogeneous user behavior is an unrealistic scenario for most applications and users and only serves as a simplification in case users of a group cannot be examined individually. If individual users in an inhomogeneous group of users use NL disproportionately often or for a long time, it may be beneficial to equip those users with an EL, so in the best case a mixed licensing is achieved. With the capacity increase (marginal capacity) of each additional NL $\left(G K_{N L}\right)$, which rises in case of an increasing group size due to the directive gain, and the cost ratio $c$, we can still find a sufficient condition in terms 


\begin{tabular}{|c|c|c|}
\hline Operation system & Description & Formulas for quality of service \\
\hline $\mathrm{M} / \mathrm{M} / \mathrm{k} / \mathrm{k}$ & $\begin{array}{l}\text { Loss system: } k \text { operating units } \\
\text { (licenses) without queue, random arrival } \\
\text { process, and negatively exponentionally } \\
\text { distributed operating time. Infinite } \\
\text { number of sources (users). }\end{array}$ & $\begin{array}{l}\text { Erlang-B-formula } \\
\text { extended-Erlang-B-formula } \\
\text { Poisson (Molina) }\end{array}$ \\
\hline $\mathrm{M} / \mathrm{M} / \mathrm{k} / \mathrm{k} / \mathrm{n}$ & $\begin{array}{l}\text { Loss system: the same as } \\
\mathrm{M} / \mathrm{M} / \mathrm{k} / \mathrm{k} \text { but with } n \text { sources. }\end{array}$ & Engset-formula \\
\hline $\mathrm{M} / \mathrm{M} / \mathrm{k}$ & $\begin{array}{l}\text { Waiting system: the same as } \mathrm{M} / \mathrm{M} / \mathrm{k} / \mathrm{k} \\
\text { but with random length of queues. }\end{array}$ & Erlang-C-formula \\
\hline arbitrary & Random distribution & Simulation \\
\hline
\end{tabular}

of the optimum in an non-homogeneous group of users: The full licensing of an non-homogeneous user group with NL reaches its optimum when the BHT of each user is lower than the ratio $\frac{G K_{N L}}{c}$. Therefore, the users must only be equal (homogeneous) in terms of this ratio.

The relation corresponds to the load at which the marginal cost of NL and EL are the same. If the load for each user and each time interval is smaller, it is not profitable to supply any user of the group with EL.

Fig. 3 shows the marginal load per user depending on the cost ratio for different group sizes (25 to 1000 users). Particularly for applications that are not permanently used and whose cost ratio EL to NL is in the normal range (between 1.25 and 2), a full licensing with NL is always optimal for large numbers of user. In this case, it is not necessary to know the user behavior in detail for determining the optimum. For smaller groups, however, the load tolerance is lower, requiring a detailed examination on the one hand and possibly leading to beneficial licensing with EL on the other hand.

\section{Determination of blocking probabilities and waiting times}

For the function $B$, which is required for the optimization, or the resulting function $S$, different formulas may be used depending on the operating system. Tab. 2 provides an overview of operating systems and formulas for calculating the quality of service, which are used in different application areas, such as dimensioning of production systems (Grundmann 2003, p. 160), database servers (Millsap and Holt 2003, p. 230), or call centers (Stolletz 2003, p. 53).

For the detailed design and definition of the formulas, we refer to the standard literature (see Bates and Gregory 2001, p. 168; Cohen 1957; Stidham 2002, p. 202 ff).
According to Parkinson (2002, p. 4), we can assume an infinite number of users as from a user base of more than 200 or a ratio of user to NL of at least 8:1, without significantly distorting the results. The extended Erlang-B-formula takes rejected requests into account which are repeated immediately. However, this effect may be neglected in the case of a very small blocking probability. For the dissolution of the formulas in terms of operating units, a numerical procedure or a pre-computed table is used in many cases (Cole 1998, p. 406; Clery 2006). An estimate of errors of the formulas mentioned here for NL operating systems is given by Järvinen et al. (2007).

\section{Risk assessment}

When using EL and NL, there is in principle no cost risk as long as, e. g., no licenses have to be purchased retrospectively due to substantial changes in user behavior. Therefore, an optimization in the LPM is initially carried out only with regard to cost issues. Changes in user behavior or load profile, e. g. by recruiting new employees, business-related or economic events (e. g. annual report, recession), affect the blocking probability and the waiting time, thus directly affecting the service quality risk.

The following example illustrates the approach. For the calculation we assume an $\mathbf{M} / \mathbf{M} / \mathbf{k} / \mathbf{k}$ operating system and the Erlang-B formula is used. All the examples were calculated using the Erlang Excel Add-in by Westbay (2007).

\section{License management at the university: Adobe Acrobat for a user group}

User groups at a university may license Adobe Acrobat Professional
7.0 either with EL for EUR 37.38 each or EUR 61.75 for each NL license (Adobe Academic Price 2007). The usage behavior within a group is not distinguished, so we just have to oppose the uniform licensing with EL to that with NL in order to determine the optimum. For example, for a group of 200 employees and a maximum license load of $L_{\tau}(P)=150$ in the $\mathrm{BH}$ (see group A, Fig. 1) it follows that all users should be equipped with NL at $Q=0,0001 \%$, which is much more expensive compared to the version with EL for EUR 7,476. For a second group with 300 employees and $L_{\tau}(P)=80$ in the $\mathrm{BH}$ (see group B, Fig. 1) the NL option with $125 \mathrm{NL}$ or EUR 7,719 is advantageous (compared to EL for EUR 11,214). The use of NL for group B reduces the total costs of 18,690 EUR for a licensing of both groups with EL by $18.7 \%$ to EUR 15,195.

This example shows how possible savings can be achieved through an adequate selection of license types. If the demands for quality of service are sufficiently high, the user hardly perceives the difference in license types. Moreover, NL allows for a greater flexibility to balance fluctuations in the number of employees since it is not necessary to purchase another license for every newcomer. NL are usually offered in the field of expensive specialized software (see Tab. 1), EL, however, are commonly used for large standard applications. Due to new trends, though, it is to be expected that flexibility of licensing standard software will increase.

\subsection{Licensing of multiple user groups with on-demand licensing}

An exclusive licensing of non-homogeneous users with EL or NL does not necessarily lead to the optimum. Since there also is the possibility of using ondemand licenses as an alternative or in addition (as license or SaaS application) in order to cover occurring peak loads and fluctuations in demand in an flexible way, the basic model is extended:

(A1') The same as A1, but providing the application for several disjoint groups $g \in G_{G e s}$ with $A_{g, G e s} \in N$ users each. $g \in G_{E L}$ refers to those groups which individually rather require EL licensing and $g \in G_{N L}$ to those which are better off with 


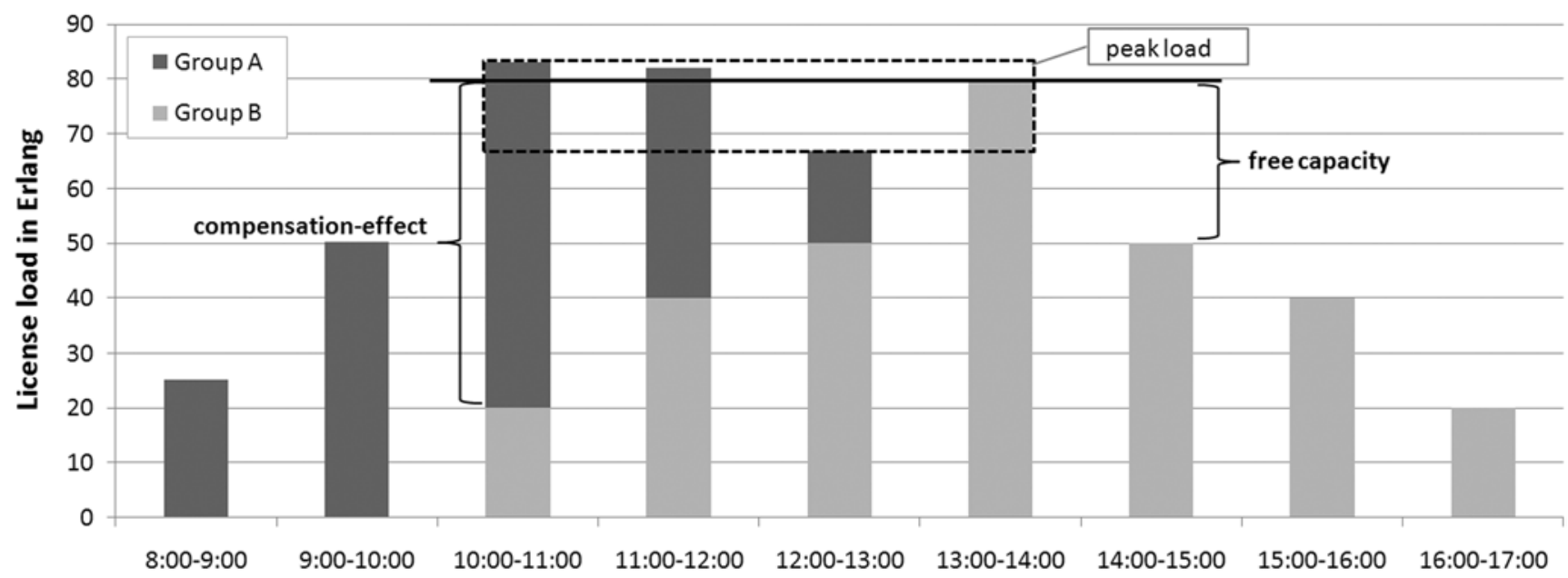

Time interval in the course of the day

Fig. 4 Load profile of a (sub-) group combination of two user groups

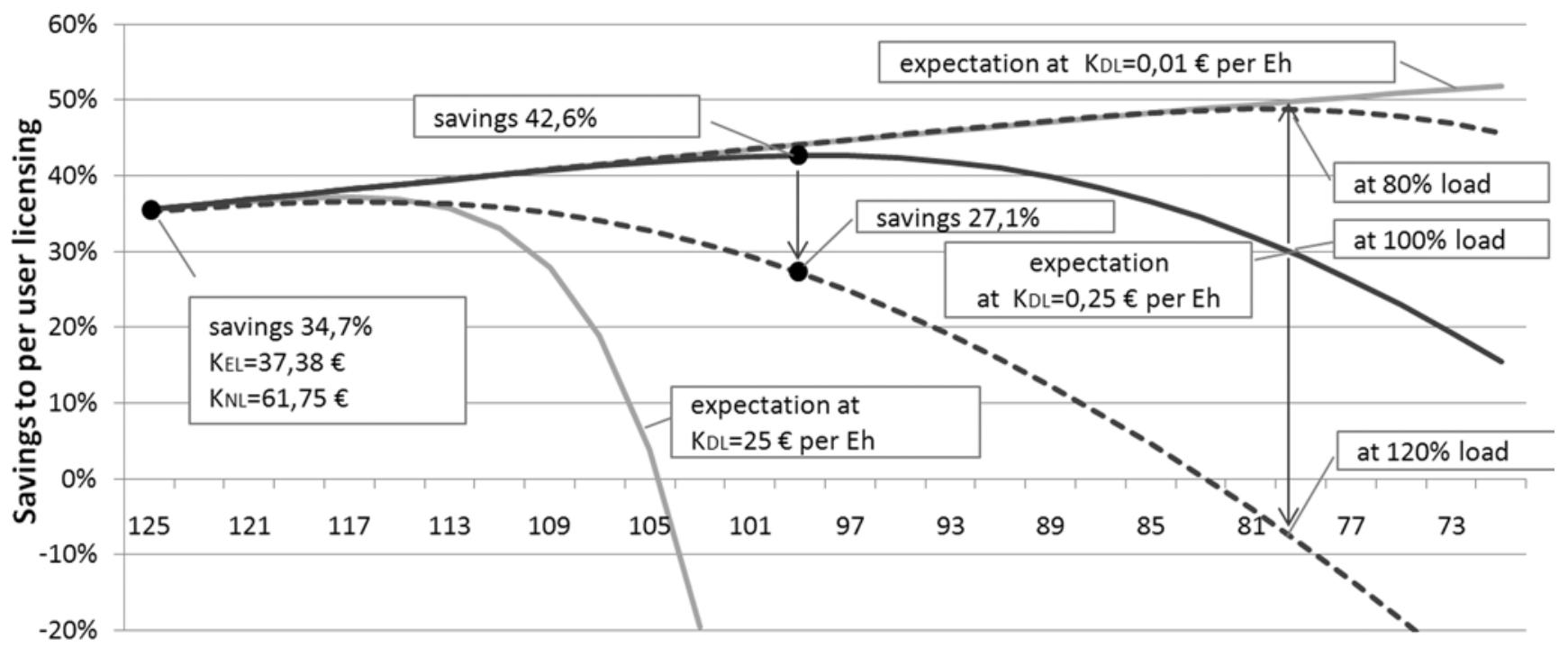

Number of concurrent licenses

Fig. 5 Expected value and risk of the savings when using DL

NL licenses. In both cases the following applies: $G_{E L} \cup G_{N L}=G_{G e s}$. The users of the groups $g \in G_{E L}$ are not distinguished in their usage behavior. In the extreme case, each user represents one group.

(A2') The same as A2, but on-demand licenses (DL) or an equivalent on-demand alternative (e. g. as SaaS application) are also available for the application, which is licensed according to the demand. The allocation of DL is carried out by a loss system. Rejected requests to NL receive DL without delay or will be diverted to the alternative. Since DL cause operating costs, we also must consider the period of application $Z$ of the users. $Z_{t} \in R^{+}$expresses how often a time interval $t$ is included within the period.
(A3') The same as A3, but now an averaged load profile $P_{g}$ for each group exists. All load profiles should be adjusted so that they have the same total length and identical time intervals.

(A5') The same as A5, but a DL additionally causes load- and time-dependent license costs for the served load capacity in the amount of $K_{D L} \in R^{+}$per Erlang-hour (Eh) (see ITU-D 2005, p. 40), considering only proportional rates because of the short-time licensing.

In addition to the directive gain, the compensation effect plays a major role with regard to licensing of several groups. The capacity of load-reduced time intervals can benefit other users without any consequences for the quality of service as long as the BHT is not increased. Compensation effects often occur when there are e. g. different core working hours caused by shift operations or different time zones in international companies. Due to the directive gain it follows that any user in a user group, which is individually licensed with $\mathrm{NL}$, continues to receive $\mathrm{NL}$ in the optimum combination of a group. If only NL-groups are available $\left(\forall g\left(g \in G_{N L}\right)\right)$, the licensing of all groups in combination with NL is optimal. If user groups $g \in G_{E L}$ exist, which individually receive EL, no statement about the optimal licensing of the group combination can be made due to the directive gain and the compensation effect. In dependence of the usage behavior or load profile, parts or even all users 


\section{COMMERZBANK}

\section{There are many new challenges for German banks. And there is one new bank for Germany.}

The new Commerzbank - your leading bank in Germany

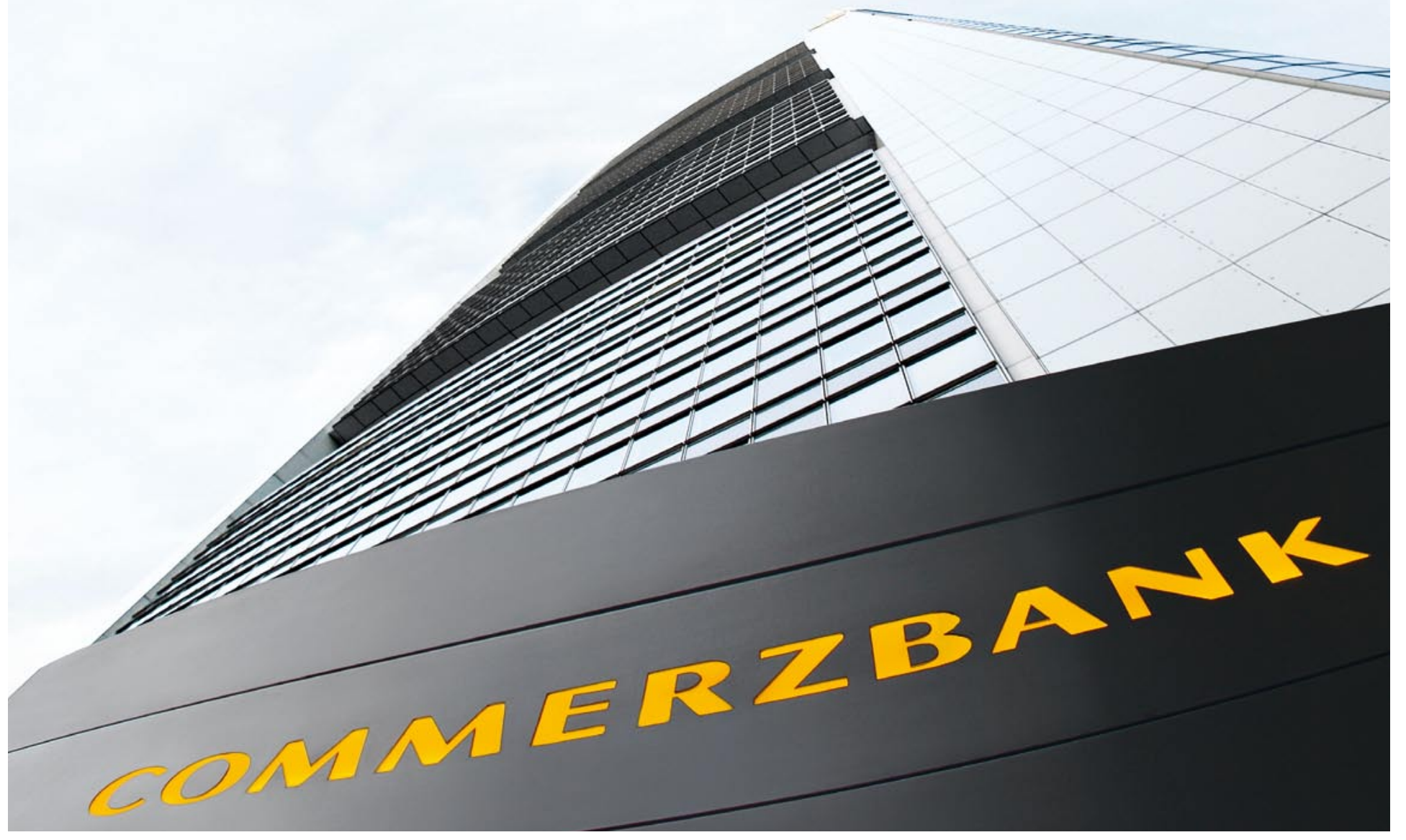

In times like these, bank customers value a partner that they can trust implicitly. A solid foundation is an equally important prerequisite for a successful partnership.

The Commerzbank can offer you this security, today and in the future. The takeover of the Dresdner Bank combines the extensive experience of both banks. Together, we will further increase our performance, and with a broad and robust business model, will prove accomplished in reacting to the changing conditions.

Thus, a new major player on the banking market emerges - and a new market leader in Germany. www.commerzbank.com

Commerzbank AG, Kaiserplatz, 60261 Frankfurt am Main, Germany 
of the group may receive NL or DL in the optimum. For these groups it therefore has to be determined how many users are optimally part of the aggregated NL load profile $P_{N L}$.

Fig. 4 shows an example of an aggregated load profile, consisting of the entire group B and a part of the users from group A (see dark bar in Fig. 1). We can identify the compensatory effect resulting from the spare capacity in the first five time intervals as well as occurring peak loads.

Besides the number of EL and NL, it is decisive for the total cost how much license load is redirected to DL. The expectation value in the time interval $t$ corresponds to the product of license load $L_{t}$, the blocking probability $B\left(L_{t}\left(P_{N L}\right), N_{N L}\right)$ and $z_{t}$. In the period $Z$ this value then has to be multiplied by the number $Z_{t}$. The sum over all time intervals $t \in T$, multiplied by the costs $K_{D L}$ makes up the DL licensing costs.

The quality of service $Q$ so far specified for NL is no longer relevant for the use of DL, since a license is available at any time. The optimization is based on the ELusing groups $A_{g}$ with $g \in G_{E L}$ and the variable number $N_{N L}^{g}$. In each group $g \in G_{E L}$, the number $A_{g, N L} \in \mathrm{N}_{0}$ with $0 \leq A_{g, N L} \leq A_{g, G e s}$ users who will receive NL or DL in the combination group needs to be determined. All group profiles $P_{g}$ of the groups $g \in G_{N L}$ and all the profiles of groups $g \in G_{E L}$ with the corresponding proportion are assigned to the aggregated load profile $P_{N L}$ of the NL or DL group.

The expected value of the total costs $E\left(K_{G e s}\right)$ are:

$E\left(K_{G e s}\right)=K_{E L} \cdot\left(\left(\sum_{g \in G} A_{g, E L}\right)^{u}+c \cdot N_{N L}^{\nu}\right)+$ $K_{D L} \cdot \sum_{t \in T}\left(z_{t} \cdot Z_{t} \cdot L_{t}\left(P_{N L}\right) \cdot B\left(L_{t}\left(P_{N L}\right), N_{N L}\right)\right)$ where:

$P_{N L}=\sum_{g \in G}\left(\frac{A_{g, N L}}{A_{g, G e s}} \cdot P_{g}\right) \quad$ and

$g \in G_{N L} \Rightarrow A_{g, N L}=A_{g, G e s} \Leftrightarrow A_{g, E L}=0$.

Moreover, it applies that:

$\operatorname{Lim}_{q \rightarrow 0} K_{G e s}=K_{E L} \cdot\left(\left(\sum_{g \in G} A_{g, E L}\right)^{u}+c \cdot N_{N L}^{\nu}\right)$,

because $B\left(L_{t}\left(P_{N L}\right), N_{N L}\right)=0$, if $N_{N L}=S\left(L_{\tau}\left(P_{N L}\right), q \rightarrow 0\right)$

and

$\operatorname{Lim}_{q \rightarrow 1} K_{\text {Ges }}=K_{E L} \cdot\left(\sum_{g \in G} A_{g, E L}\right)^{u}$

$+K_{D L} \cdot \sum_{t \in T}\left(z_{t} \cdot Z_{t} \cdot L_{t}\left(P_{N L}\right)\right)$.

because $N_{N L}=S\left(L_{\tau}\left(P_{N L}\right), q \rightarrow 1\right)=0$.

This means that for a very small $q$ the number of NL is so high that no access will be blocked and therefore no DL are needed. However, if no NL $(q=1)$ is used, the entire load that is not covered by the EL will be compensated by DL. For a single homogeneous group, the effect of directive gain also means that a mixed licensing, containing EL, makes no sense and in the optimum therefore either EL or a combination of NL and DL will be used.

Due to the transformation of the service quality risk into a cost risk, the total costs identified in section 3.1 are restricted in terms of their comparability for the presented case. The objective function for a homogeneous group of users is:

$$
\begin{aligned}
& E\left(K_{\text {Ges }}^{*}\right)=\operatorname{Min}\left\{K_{E L} \cdot A_{\text {Ges }}^{u} ;\left(K_{N L} \cdot N_{N L}^{\nu}+K_{D L} \cdot \sum_{t \in T}\right.\right. \\
& \left.\left.\left(z_{t} \cdot Z_{t} \cdot L_{t}\left(P_{N L}\right) \cdot B\left(L_{t}\left(P_{N L}\right), N_{N L}\right)\right)\right)\right\} .
\end{aligned}
$$

Although the calculation is restricted to the groups $g \in G_{E L}$, possibly heuristics have to be used for many and large groups, which e. g. gradually enlarge or reduce the size of the other groups.

\subsection{Risk assessment}

Contrary to the model without DL, there is no quality of service risk left; however, cost risk which may result from the uncertainty in the load profile $P_{N L}$ still exists. If mainly EL and NL are used, the expected value of the DL licensing costs is reduced and load fluctuations have only fractional effects. In the case of few EL and NL the expected value and the risk of DL costs are accordingly increased because more load is redirected to DL.

Fig. 5 shows for the example of Adobe Acrobat how the expected value and risk of the savings change in dependence on the number of NL (black solid line), a load variation of $20 \%$ (dashed lines), and varying DL cost (gray lines). When reaching $125 \mathrm{NL}$ in the example, even in the case of load fluctuations additional DL are hardly needed. The expected value of the cost savings rises to a maximum of $42.6 \%$ at 99 NL. Load fluctuations have a higher impact on costs when the number of NL decreases, causing an increase of the risk that the expected savings might not be achieved. This curve, initially upward, is typical, as "expensive" DL are worthwhile for the compensation of at least small peak loads.

In the case of a decreasing number of NL, the licensing load and thus the marginal cost of the DL increase until in the optimum the marginal costs of NL are reached or both have become equal. At very low DL costs or very rare use of an application, it is in the extreme case possible to deploy DL for licensing only. If next to DL only EL are available, the deployment is profitable if users or systems are only temporarily used within the considered period.

\section{License management at the university: Adobe Acrobat with on- demand licenses for multiple user groups}

Licensing of group A and B with NL only reduces the total cost at $Q=0,0001 \%$ by $23.0 \%$ to EUR 14,388 due to the directive gain. For the use of DL we assume that the use of Adobe Acrobat via the planned SaaS platform Acrobat.com costs EUR 0.25 per Eh and that the period is 600 days.

The analysis shows that the optimum of 84 users in group A and all users in Group B together receive 99 NL (see Fig. 5). The expected total cost is EUR 10,729, the saving is $42.6 \%$. However, in case of e. g. $20 \%$ of additional load, the savings would decrease to $27.1 \%$.

The example shows that by integrating use-intensive users in a NL group, EL costs can be saved. Especially in international organizations with large groups and deferred load profiles, a high quality of service level can be guaranteed by means of relatively few NL.

However, in case of business critical applications, the uncertainty with regard to the quality of service at peak loads leads to the fact that IT managers prefer safe EL or significantly increase the number of NL. In combination with the NL, the use of DL can be used to reduce the quality of service risk and utilize further possible savings as long as the availability of the DL can be ensured.

In the field of server software, DL are largely used for business critical applications to temporarily cover peak loads by adding capacity such as processors or virtual server systems. In the case of application software, however, DL are still rarely used despite these advantages. One reason is that so far no standardized procedures for the allocation and collection of required DL exist. Each manufacturer uses 
an individual licensing system that ranges from independent hardware dongles to online access via the internet, considerably increasing the administrative effort for many applications. Another issue, particularly affecting SaaS applications, can be identified in the associated risks in terms of data security and availability if applications and data (e. g. confidential management reports, customer data or transaction information) are provided beyond the boundaries of the individual company. Many IT managers have been sensitized e. g. due to the recent data losses in SaaS applications of large enterprises and government authorities, and are therefore not willing to take an incalculable risk. Finally, they even avoid DL because of the cost risk, which, however, could be limited by appropriately designed pricing models. Thus, in addition to the usual upgrade rights, we could think of variable quotas for DL as part of a license bundling with EL or NL, which would reduce both quality of service and cost risk.

\section{Summary of results and outlook}

The growing availability of dynamic and flexible on-demand licensing concepts opens additional opportunities for cost reduction, which may further improve the savings of avoiding an over licensing.

The presented model allows an evaluation and comparison of licensing decisions in an objectified way. We showed that for a large user group in most cases a mixed licensing of EL and NL does not prove to be economically efficient even in case of non-homogeneous user behavior. In the second part of the contribution, we examined the situation with several user groups and a demand-oriented licensing type. Especially in international business NL are profitable even for use-intensive user groups due to the time shift of the user profiles (such as e. g. special software in investment banking). However, this requires enterprise-wide license management and the possibility of cross-country licensing.

To cover peak loads and demand fluctuations, DL can reduce quality of service risks and costs. Despite these advantages, this license type so far is rarely used for application software because besides the associated cost and safety risks, the still small amount of offers prevents a comprehensive deployment.
Further research is needed with regard to the question of how appropriate user groups can be identified within the company. Moreover, there is the challenge of avoiding malpractice by the user (e. g. the permanent blocking of NL beyond the actual usage time or the unnecessary use of DL). This requires the development of incentive models which may for instance be used to prompt user groups to work outside of peak loads and only systematically use DL applications.

As the subject of license portfolio management has so far received little attention in scientific literature and the relevance of software licenses in the company is steadily increasing, this contribution marks a first focus point for subsequent work.

\section{References}

Bates RJ, Gregory DW (2001) Voice and data communications handbook, $4^{\text {th }}$ edn. McGraw-Hill Osborne Media, New York

BDSG (2003) Bundesdatenschutzgesetz in der Fassung der Bekanntmachung vom 14. Januar 2003. http://bundesrecht.juris.de/bundesrecht/bdsg_1990/gesamt.pdf. Accessed 200805-01

Bensberg F, Reepmeyer J (1994) Lizenzmanagement in lokalen Netzwerken - rechtliche Grundlagen, organisatorische Konzepte, Softwarewerkzeuge. WIRTSCHAFTSINFORMATIK 36(6):591-599

Bose S (2002) An introduction to queueing systems. Springer, Heidelberg

BSA (2007) BSA führt weltweit größte Antipiraterie-Aktion durch: Internationales Medienunternehmen lernt den Preis unlizenzierter Software kennen. http://www.bsa.org/germany/presse/ newsreleases/BS097-16.cfm. Accessed 200710-12

Buhl HU (1993) Finanzanalyse von Entscheidungsalternativen bei der Software-Vertragsgestaltung. Zeitschrift für betriebswirtschaftliche Forschung 45:911-932

CIO (2003) Lizenz zum Entrümpeln. ClO Magazin. http://www.cio.de/markt/804236/index.html. Accessed 2007-06-16

Clery R (2006) Traffic table by Roger Clery Roosevelt University. http://cs.roosevelt.edu/rutelecom/331/Btable.htm. Accessed 2007-06-16

Cohen JW (1957) The generalized Engset formulae. Philips Telecomm 18:158-170

Cole M (1998) Telecommunications. Prentice Hall, New Jersey

Gartner (2001) IT asset management: reduce costs and minimize risks. Gartner Group. http:// www.gartner.com/DisplayDocument?ref=g_se arch\&id $=345044 \&$ subref $=$ simplesearch. Accessed 2007-06-16

Grundmann W (2003) Operations Research. Formeln und Methoden. Vieweg, Wiesbaden

IDC (2007) Fourth annual BSA And IDC global

\section{Abstract}

Daniel Gull, Alexander Wehrmann

Optimized Software Licensing Combining License Types in a License Portfolio

Although software licenses usually range among the most expensive items within the IT budget, they still lack the necessary attention by many companies. Therefore, most companies inadequately have implemented their software asset management neglecting further potential for cost reduction, which can be obtained by optimizing the use of different license types. This paper shows how possible savings can be realized by combining different types of licenses in a license portfolio. The model presented is based on the most common license types considering different user groups as well as their behavior. Additionally to cost risks, the risk of service quality is also taken into consideration. The following examples illustrate the model's high relevance and show how it can be applied in practice.

Keywords: License portfolios, License types, Software licensing, License management 
software piracy study. http://w3.bsa.org/ globalstudy. Accessed 2007-06-16

ITU-D (2005) Handbook teletraffic engineering. ITU-D Group 2. http://www.tele.dtu.dk/ teletraffic/handbook/telehook.pdf. Accessed 2006-08-16

Järvinen J, Johnsson M, Leipälä T, Murtojärvi M, Nevalainen $O$ (2007) Determining the proper number and price of software licenses. IEEE Transactions on Software Engineering 33(5):305-315

Junk V, Warnecke H (2002) Handbuch für Telekommunikation, $2^{\text {nd }}$ edn. Springer, Heidelberg KMPG (2002) Lizenzmanagement in deutschen Unternehmen. http://www.kpmg.de/library/ pdf/020620_Lizenzmanagement_in_ deutschen_Unternehmen_de.pdf. Accessed 2006-08-16

Macrovision (2006) Key trends in software pricing and licensing - A survey of software industry executives and their enterprise customers. Macrovision. http://www.softsummit.com/ softsummit_knowledge_library_industry_ reports.shtml. Accessed 2007-05-12

Mendel T, Takahashi S (2007) 2007 Enterprise IT budget outlook: Europe. Forrester Research. http://www.forrester.com/Research/ Document/Excerpt/0,7211,41668,00.html. Accessed 2007-09-12

Microsoft (2007) Studie 2007: Software- und Lizenzmanagement in deutschen Unternehmen. http://www.microsoft.com/germany/ mittelstand/lizenzen/software-assetmanagement-downloads.mspx. Accessed 2007-09-12

Millsap CV, Holt JL (2003) Optimizing Oracle performance, $1^{\text {st }}$ edn. O'Reilly, Cologne

Müller P, Nasterlack S, Schwarze L (2006) Professionelles IT Lizenzmanagement - die Herausforderung für die Zukunft. Information Management \& Consulting 21:14-19

Parkinson R (2002) Traffic engineering techniques in telecommunications. http://www. infotel-systems.com/Downloads/ TrafEngWhitePaper.pdf. Accessed 2007-05-12

RegTP (2005) Ein analytisches Kostenmodell für das Breitbandnetz. http://www. bundesnetzagentur.de/media/archive/886.pdf. Accessed 2007-05-12

Rey RF (1983) Engineering and operations in the Bell system. AT\&T Bell Laboratories. Murray Hill, New York

Sedlmeier T (2006) Softwarelizenzmodelle aus rechtlicher Sicht - Wissenswertes für IT-Projektleiter. Information \& Management Consulting 21:10-12

Stache U, Zimmermann W (2001) Operations Research, $10^{\text {th }}$ edn. Oldenbourg, München

Stapperfend T (1991) Die steuer- und bilanzrechtliche Behandlung von Software. Dr. Otto Schmidt, Cologne

Stidham S (2002) Analysis, design, and control of queueing wystems. Operations Research 50(1):197-216

Stolletz R (2003) Performance analysis and optimization of inbound call centers. Springer, Heidelberg

Taha HA (1992) An introduction to operations re- search, $5^{\text {th }}$ edn. Prentice Hall, New Jersey

Tran-Gia P (2005) Einführung in die Leistungsbewertung und Verkehrstheorie, $2^{\text {nd }}$ edn. Oldenbourg, München

Westbay (2007) Erlang for Excel - Add-in for Microsoft Excel. http://www.erlang.com/excel. html. Accessed 2007-03-13

Wisotzky H (2006) Implementierung des LizenzManagements im Unternehmen. Information Management \& Consulting 21:6-9

Znidarsic D (2006) Waste not, want not - Software license tracking and successful vendor relations. Data Center Magazine 2:43-46

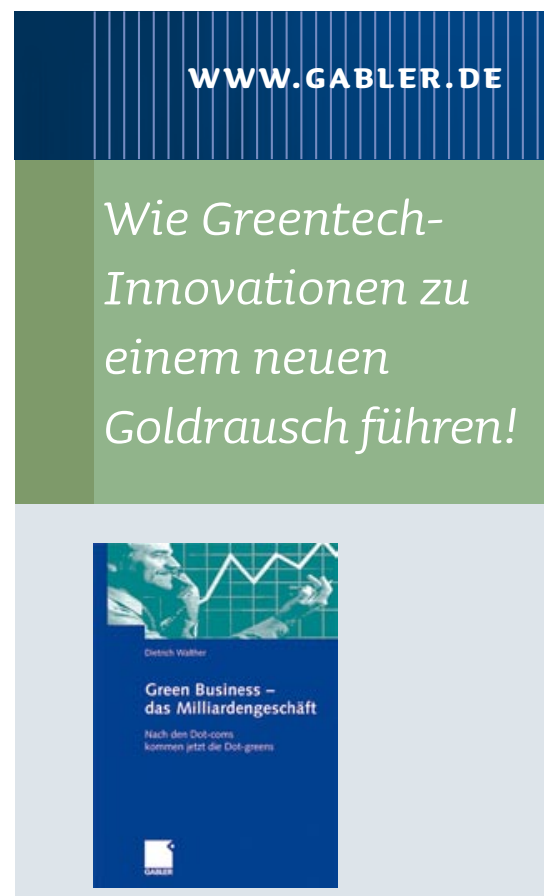

Walther, Dietrich

Green Business -

das Milliardengeschäft

Nach den Dot-coms kommen jetzt die Dot-greens

Unter Mitarbeit von Wolf D. Hartmann, 2009. 220 S. Br.

EUR 39,90

ISBN 978-3-8349-1273-2

Fax $+49(0) 611.7878-420$

Ja, ich bestelle

Expl. Green Business -

das Milliardengeschäf

ISBN 978-3-8349-1273-2

EUR 39,90 zuzügl. Versand EUR 3,32

Name, Vorname

Firma

Straße (bitte kein Postfach)

PLZ | Ort

Datum | Unterschrift 22109002

KOMPETENZ IN

SACHEN WIRTSCHAFT

Änderungen vorbehalten Erhältlich im Buchhand oder beim Verlag.

Dr. Ralf Birkelbach, Albrecht F. Schirmache AG Wiesbaden HRB 9754. 